\title{
Association of the RASS Score With Intensity of Symptoms, Discomfort, and Communication Capacity in Terminally III Cancer Patients Receiving Palliative Sedation: Is RASS an Appropriate Outcome Measure?
}

Kengo Imai ( $\nabla$ k.imai@sis.seirei.or.jp )

Seirei Mikatahara General Hospital

Tatsuya Morita

Seirei Mikatahara General Hospital

Naosuke Yokomichi

Seirei Mikatahara General Hospital

Masanori Mori

Seirei Mikatahara General Hospital

Akemi Shirado Naito

Miyazaki Medical Association Hospital

Toshihiro Yamauchi

Seirei Mikatahara General Hospital

Hiroaki Tsukuura

Akari Home Care Clinic

Yu Uneno

Kyoto University

Satoru Tsuneto

Kyoto University

Satoshi Inoue

Seirei Mikatahara General Hospital

Research Article

Keywords: Palliative sedation, measurement, Richmond Agitation-Sedation Scale, Support Team Assessment Schedule, Discomfort Scale for Dementia of Alzheimer Type, Non-communicative Patient's Pain Assessment Instrument, Communication Capacity Scale

Posted Date: October 6th, 2021 
DOl: https://doi.org/10.21203/rs.3.rs-929226/v1

License: (c) (1) This work is licensed under a Creative Commons Attribution 4.0 International License. Read Full License

Version of Record: A version of this preprint was published at Palliative Medicine Reports on April 1st, 2022. See the published version at https://doi.org/10.1089/pmr.2021.0087. 


\section{Abstract}

\section{Background}

Palliative sedation is sometimes needed for refractory symptoms, and the Richmond Agitation-Sedation Scale (RASS) is one of the key measures. The primary aim of this study was to explore the association between the RASS score and degree of distress quantified by other measures: Support Team Assessment Schedule, item 2 (STAS), Discomfort Scale for Dementia of Alzheimer Type (Discomfort Scale), and Noncommunicative Patient's Pain Assessment Instrument (NOPPAIN), as well as a communication capacity measured by the Communication Capacity Scale, item 4 (CCS).

Methods

This was a prospective observational study on terminally ill cancer patients who received continuous infusion of midazolam to relieve refractory symptoms in a palliative care unit of a designated cancer hospital. Primarily responsible palliative care physicians rated RASS, Discomfort Scale, NOPPAIN, and CCS just before starting infusion, and 1 hour, 4 hours, 24 hours, and 48 hours after midazolam infusion, and ward nurses rated STAS at the same time. Since the ward nurses regularly evaluated STAS to titrate midazolam during palliative sedation as routine work, we regarded STAS rated by ward nurses as a standard of distress measure.

Results

A total of 249 assessments were performed for 55 patients. The RASS score was moderately to highly associated with symptom intensity measured by STAS, discomfort measured by the Discomfort Scale, and pain measured by NOPPAIN ( $r=0.63$ to 0.73 ). On the other hand, communication capacity measured by CCS is not parallel with the RASS score, and demonstrated a valley-shape. In 82 assessments with RASS of -1 to $-3,11$ patients (13\%) were regarded as having physical symptoms of STAS of 2 or more.

\section{Conclusions}

RASS can roughly estimate physical distress in patients receiving palliative sedation, but a measure to more precisely quantify the symptom experience is needed.

\section{Background}

Some patients experience intense symptoms refractory to intensive palliative care before death, and palliative sedation is used [1-4]. There are many observation studies about the efficacy of palliative sedation, but the variability of outcome measures make interpretation difficult $[1,5-8]$.

While the Critical Care Pain Observation Tool (CPOT) is recommended as a measure of pain in this setting $[9,10]$, the Richmond Agitation-Sedation Scale (RASS) is one of the key measures in clinical studies about palliative sedation, or terminal delirium (the most common indication of palliative 
sedation) [11-16]. Originally, RASS measures the degree of agitation and sedation [17-20], and understanding potential associations of the RASS scores and other measures is valuable to determine whether RASS can be a general indicator of palliative sedation. Additionally, maintaining communication capacity is one of the important goals in palliative care [21,22], but information about the relationship between the RASS score and communication capacity is lacking.

The primary aim of this study was thus to explore the association between the RASS score and degree of distress using other measures: Support Team Assessment Schedule (STAS), item 2 [23, 24], Discomfort Scale for Dementia of Alzheimer Type (Discomfort Scale) [25-27], and Non-communicative Patient's Pain Assessment Instrument (NOPPAIN) [28, 29], as well as a communication capacity measured by the Communication Capacity Scale (CCS), item 4 [30-33]. The second aims were physical symptoms according to each RASS level to reveal distress that was undetected by RASS, and factor analyses using items of the Discomfort Scale and NOPPAIN to explore underlying structure on each scale.

\section{Subject And Methods}

This was a prospective observational study on terminally ill cancer patients who received the continuous infusion of midazolam to relieve refractory symptoms. Patients admitted to a palliative care unit of a 934-bed designated cancer hospital, the Seirei Mikatahara General Hospital, Japan, were consecutively enrolled between November 2015 and March 2017. The PCU had 27 beds, and 5 full-time palliative care specialists were responsible for inpatient care. The ethical and scientific validities of this study were verified by the institutional review board (IRB) of the hospital. We used an opt-out method rather than obtaining written informed consent following the decision of the IRB.

Primarily responsible physicians rated RASS, Discomfort Scale, NOPPAIN, and CCS just before starting infusion, and 1 hour, 4 hours, 24 hours, and 48 hours after midazolam infusion; and ward nurses rated STAS at the same time. Since the ward nurses regularly evaluated STAS to titrate midazolam during palliative sedation as routine work, we regarded STAS rated by ward nurses as a standard of distress measure. Each patient underwent a maximum of 5 assessments.

\section{Outcome measures}

Richmond Agitation-Sedation Scale (RASS)

RASS is one of the most commonly used scales to determine the sedation level, and it measures the severity of agitation and sedation with a score of +4 to -5 : +4 : combative, +3 : very agitated, +2 : agitated, +1: restless, 0 : alert and calm, -1 : drowsy, -2 : light sedation, -3 : moderate sedation, -4 : deep sedation, and -5 : unarousable $[17,18]$. We used the modified RASS that was revised from the original version for use in a palliative care setting: 1 ) removal of the description about the ventilator, 2) addition of further description to clarify the meaning of RASS +1 , and 3 ) deletion of physical stimulation (rubbing sternum) in the procedure for RASS assessment $[19,20]$. 
The Support Team Assessment Schedule is the validated measure of comprehensive assessment for palliative care patients $[23,24]$. To measure the intensity of symptoms, we decided to use the highest individual symptom score in item 2 (symptom control) of STAS: 0 : none, 1: occasional or grumbling single or few symptoms, 2: moderate distress, 3: severe symptoms present often, and 4: severe and continuous overwhelming symptoms. Patients with unconsciousness and no detectable symptoms were rated as 0 .

Discomfort Scale for Dementia of Alzheimer Type (Discomfort Scale)

The Discomfort Scale is a measure to rate the discomfort level of patients with dementia of Alzheimer type, consisting of 9 items from 0 (none) to 3 (severe), with a possible range of 0 to 27 [25-27]. A higher score indicates greater discomfort of patients.

Non-communicative Patient's Pain Assessment Instrument (NOPPAIN)

NOPPAIN is a measure to rate the pain level of non-communicative patients, consisting of 6 items, from 0 (lowest possible) to 5 (highest possible), with a possible range of 0 to 30 [28]. A higher score indicates greater pain of patients, and the scores of NOPPAIN are associated with other objective pain assessment tools, such as the Abbey Pain Scale and Pain Assessment in Advanced Dementia Scale (PAINAD) [29].

Communication Capacity Scale (CCS)

CCS was developed to estimate the level of communication capacity of terminally ill patients, and we used item 4 , with a score of 0 : voluntary and explicit communication of complex contents; 1 : voluntary and explicit communication of simple contents; 2 : involuntary or inexplicit communication of simple contents; and 3: unable to communicate [30-33].

Statistics analyses

Patient characteristics and outcome measures were summarized using descriptive analyses. To investigate the potential association among the scales, correlation coefficients were calculated using Pearson's coefficients. To explore the difference in scores of STAS, Discomfort Scale, NOPPAIN, and CCS according to each RASS level, we plotted the means with standard errors and compared the scores using ANOVA. A post-hoc test was not used. Furthermore, we calculated how many patients were rated as distressed based on STAS for each RASS score to identify the proportion of underestimation of distress by RASS, i.e., RASS of -1 or less but STAS of 2 or more. Finally, to explore the underlying factor structure, all items of the Discomfort scale and NOPPAIN were analyzed using promax rotation. The number of factors was determined using the scree plot and an eigen value of 1 . We performed a sensitivity analysis for each evaluating physician. The data were analyzed using SPSS version 26 (IBM, Tokyo, Japan, 2019).

\section{Results}


A total of 249 assessments were performed for 55 patients. Patient characteristics are summarized in Table 1. Palliative sedation was provided as a form of proportional sedation in 43 (78\%) and continuous deep sedation in 12 patients (22\%). Chief target symptoms were dyspnea in 38 and delirium in 27 patients. A median of 3 assessments were performed for each patient. A total of 7 physicians provided assessments $(102,59,31,25,11,9$, and 6 for each). The difference in assessments numbers was mainly associated with the numbers of patients per physician and the physicians' length of service. Sensitivity analyses of data except for one physician who enrolled patients the most frequently generated essentially the same results. 
Table 1

Background of the patients $(\mathrm{N}=55)$

\begin{tabular}{|ll|}
\hline & $\mathrm{n}(\%)$ \\
\hline Age (years, mean and SD) & $65.4(13.2)$ \\
\hline Sex & \\
\hline Male & $34(62 \%)$ \\
\hline Female & $21(38 \%)$ \\
\hline Primary tumor sites & $15(27 \%)$ \\
\hline Lung & $9(16 \%)$ \\
Pancreas, liver, bile ducts & $8(15 \%)$ \\
Colon and rectum & $6(11 \%)$ \\
Soft tissues & $4(7.3 \%)$ \\
Stomach and esophagus & $4(7.3 \%)$ \\
Kidney, urinary tracts, bladder, prostate & $3(5.5 \%)$ \\
\hline Uterus and ovary & $2(3.6 \%)$ \\
Breast & $2(3.6 \%)$ \\
\hline Head and neck & $2(3.6 \%)$ \\
\hline Others & $38(69 \%)$ \\
\hline Sedation types & $27(49 \%)$ \\
\hline Proportional sedation & $8(15 \%)$ \\
\hline Continuous deep sedation & $4(7.3 \%)$ \\
\hline Time periods of sedation (hours, median and ranges) & $3(5.5 \%)$ \\
\hline Target symptom & $43(78 \%)$ \\
\hline Dyspnea & $12(22 \%)$ \\
\hline Delirium & \\
\hline Pain & \\
\hline Nausea/vomiting & \\
\hline
\end{tabular}


Table 2 summarizes mean and median values: -2.59 and -3 (RASS); 0.76 and 0 (STAS); 6.20 and 3 (Discomfort Scale); 2.40 and 0 (NOPPAIN); and 2.61 and 3 (CCS), respectively.

Table 2

Distributions of the score of the RASS, STAS, Discomfort Scale, NOPPAIN, and CCS

\begin{tabular}{|llll|}
\hline & $\begin{array}{l}\text { Mean } \\
\text { (SD) }\end{array}$ & $\begin{array}{c}\text { Median } \\
\text { (range) }\end{array}$ & $\begin{array}{l}\text { Distributions } \\
\text { N (\%) }\end{array}$ \\
\hline RASS & -2.59 & $-3(-5,3)$ & $-5,61(24 \%) ;-4,56(22 \%) ;$ \\
& & $-3,32(13 \%) ;-2,31(12 \%) ;$ \\
& & $-1,19(7.4 \%) ; 0,3(1.2 \%) ;$ \\
& & $+1,35(14 \%) ;+2,11(4.3 \%) ;$ \\
& & $+3,1(0.4 \%) ;+4,0(0 \%)$
\end{tabular}

The RASS score was moderately to highly associated with physical symptoms measured by STAS, discomfort measured by the Discomfort Scale, and pain measured by NOPPAIN ( $r=0.63$ to 0.73 ; Table 3 ). Figure 1 demonstrates a significant difference in the scores of the STAS, Discomfort Scale, and NOPPAIN according to each RASS level (all $\mathrm{P}<0.001$ ). On the other hand, communication capacity measured by CCS was not parallel with RASS, and demonstrated a valley-shape: the CCS score was lowest (normal communication capacity) in patients with a RASS score of 0 , and increased in both directions. 
Table 3

Correlations between the RASS score and the STAS, Discomfort Scale, and the NOPPAIN

\begin{tabular}{|lllll|}
\hline & RASS & STAS & Discomfort Scale & NOPPAIN \\
\hline RASS & 1.0 & 0.72 & 0.73 & 0.65 \\
\hline STAS & - & 1.0 & 0.72 & 0.64 \\
\hline Discomfort Scale & - & - & 1.0 & 0.63 \\
\hline NOPPAIN & - & - & - & 1.0 \\
\hline Legend for Table 3 & & & \\
\hline Pearson's correlation coefficients were described. All P values were <0.001 & \\
\hline $\begin{array}{l}\text { RASS: Richmond Agitation-Sedation Scale; STAS: Support Team Assessment Schedule, item 2 for any } \\
\text { symptoms; Discomfort Scale: Discomfort Scale for Dementia of Alzheimer Type; NOPPAIN: Non- } \\
\text { communicative patient's pain assessment Instrument; CCS: Communication Capacity Scale, item 4 }\end{array}$ \\
\hline
\end{tabular}

In 82 patients with RASS of -1 to $-3,11$ patients $(13 \%)$ were regarded as having physical symptoms of STAS of 2 or more (Table 4), while in 117 patients with a RASS score of -4 to $-5,2$ patients $(1.7 \%)$ were rated as STAS of 2 or more. 
Table 4

Patients with physical symptom using the STAS of 2 or more according to each RASS level STAS of 2 or more

\begin{tabular}{|llll|}
\hline & $N$ & $\%$ & $95 \% \mathrm{Cl}$ \\
\hline RASS & & & \\
\hline+2 or more $(n=12)$ & 9 & 75 & $46-91$ \\
\hline$+1(n=35)$ & 23 & 66 & $49-79$ \\
\hline $0(n=3)$ & 2 & 67 & $21-94$ \\
\hline$-1(n=19)$ & 3 & 16 & $5-38$ \\
\hline$-2(n=31)$ & 5 & 16 & $7-33$ \\
\hline$-3(n=32)$ & 3 & 9.4 & $3-24$ \\
\hline$-4(n=56)$ & 2 & 3.6 & $1-12$ \\
\hline-5 (n=61) & 0 & 0 & $0-6$ \\
\hline Legend for Table 4 & & & \\
\hline The numbers of the patients were described. & & & \\
\hline Cl: Confidence Interval; RASS: Richmond Agitation-Sedation Scale; STAS: Support Team Assessment \\
\hline Schedule, item 2 for any symptoms
\end{tabular}

Factor analyses using all items of the Discomfort Scale and NOPPAIN identified 3 factors (Table 5): distressed facial expression and restlessness (Factor 1), verbal expression of discomfort (Factor 2), and pain-avoiding behavior (Factor 3). 
Factor structure of the Discomfort Scale and NOPPAIN

\begin{tabular}{|lccc|}
\hline & Factor 1 & Factor 2 & Factor 3 \\
\hline Content facial expression (Discomfort Scale, reversed) & .942 & -.126 & -.049 \\
\hline Frown (Discomfort Scale) & .914 & -.006 & -.020 \\
\hline Sad facial expression (Discomfort Scale) & .884 & .007 & -.086 \\
\hline Fidgeting (Discomfort Scale) & .863 & -.064 & .009 \\
\hline Relaxed body language (Discomfort Scale, reversed) & .849 & -.106 & -.088 \\
\hline Restlessness (NOPPAIN) & .817 & .038 & .029 \\
\hline Frightened facial expression (Discomfort Scale) & .789 & .027 & .070 \\
\hline Pain face (NOPPAIN) & .763 & .131 & -.013 \\
\hline Tense body language (Discomfort Scale) & .708 & .022 & .255 \\
\hline Noisy breathing (Discomfort Scale) & .619 & .136 & -.014 \\
\hline Pain words (NOPPAIN) & -.169 & 1.00 & -.045 \\
\hline Pain noises (NOPPAIN) & .031 & .888 & .059 \\
\hline Negative vocalization (Discomfort Scale) & .375 & .622 & -.041 \\
\hline Bracing (NOPPAIN) & -.036 & -.035 & .830 \\
\hline Rubbing (NOPPAIN) & -.028 & .014 & .759 \\
\hline Legend for Table 5 & & & \\
\hline $\begin{array}{l}\text { Factor loadings were described. Factor 1: distressed facial expression and restlessness, Factor } 2: \\
\text { verbal expression of discomfort, Factor 3: pain-avoiding behavior }\end{array}$ & & & \\
\hline $\begin{array}{l}\text { Discomfort Scale: Discomfort Scale for Dementia of Alzheimer } \\
\text { patient's pain assessment Instrument }\end{array}$ & & & \\
\hline 1b) Discomfort measured by the Discomfort Scale & & & \\
\hline
\end{tabular}

\section{Discussion}

This is the first study to explore the association between the RASS level and degree of distress and pain as well as communication capacity in terminally ill cancer patients receiving palliative sedation.

One of the important findings of this study was that RASS scores were generally well-correlated with other measures of physical discomfort, including intensity of physical symptoms measured by STAS, discomfort measured by Discomfort Scale, and pain measured by NOPPAIN. But a small number of 
patients were rated as having physical symptoms among those with a RASS score of -1 to -3 . This result may be because, in the last few days, a patient often experiences a mixture of symptoms including agitated delirium, pain, dyspnea, general discomfort, and other physical symptoms. Another interpretation is that measurement tools cannot intrinsically differentiate each symptom in patients with delirium or conscious disturbance in their final days. Existing studies have indicated that, although behavior pain scales are valid in nonverbal critically ill patients and dementia patients [34,35], their validity is questionable for patients with delirium, especially agitated delirium [36-38]. Similarly, Respiratory Distress Observation Scale (RDOS), a behavior dyspnea scale for patients unable to self-report about dyspnea, is valid in palliative care patients [39,40], its validity is unknown for patients with agitated delirium and a mixture of physical symptoms. The finding that distressed facial expression and restlessness were collapsed into one category by factor analyses suggests that symptoms of delirium and pain often overlap.

Of note is that the RASS score was not linearly associated with the communication capacity. This is logical based on the definition of RASS, and thus use of the mean RASS score as an indicator of the communication capacity should be avoided, and the percentages of patients with a RASS score of 0 or the concurrent use of measures to quantify the communication capacity is reasonable.

The limitations of this study include: a single-institution study, the same patients were evaluated multiple times, and the same physician rated RASS, Discomfort Scale, and NOPPAIN.

\section{Conclusions}

In conclusion, the RASS score is generally associated with other discomfort measures and can be used to roughly estimate physical distress in patients receiving palliative sedation. But some patients may have distress undetectable by RASS, and a measure to more preciously quantify the symptom experience of patients is needed. Modification of RASS to evaluate discomfort in addition to agitation, or the concurrent use of agitation and other symptom measures is promising.

\section{Abbreviations}

RASS: Richmond Agitation-Sedation Scale; STAS: Support Team Assessment Schedule; Discomfort Scale: Discomfort Scale for Dementia of Alzheimer Type; NOPPAIN: Non-communicative Patient's Pain Assessment Instrument; CCS: Communication Capacity Scale; CPOT: Critical Care Pain Observation Tool; PAINAD: Pain Assessment in Advanced Dementia Scale; ANOVA: analysis of variance; RDOS: Respiratory Distress Observation Scale

\section{Declarations}

\section{Ethics approval and consent to participate}


We conducted this study following the ethical standards of the Helsinki Declaration and the ethical guidelines for medical and health research involving human subjects presented by the Ministry of Health, Labour, and Welfare in Japan, and was verified by the Institutional Review Board (IRB) of Seirei Mikatahara General hospital. We used an opt-out method rather than obtaining written informed consent following the decision of the IRB.

\section{Consent for publication}

Not applicable.

\section{Availability of data and materials}

The datasets used and/or analysed during the current study are available from the corresponding author on reasonable request.

\section{Competing interests}

The authors declare that they have no competing interests.

\section{Funding}

This work was supported by JSPS KAKENHI Grant Number JP19H03869. The funder provided no role within conception or design of the work, the acquisition, analysis, interpretation of data and drafting manuscript.

\section{Authors' contributions}

$\mathrm{KI}, \mathrm{TM}, \mathrm{NY}, \mathrm{MM}$, and ST have contributed to the conception and design of the work. KI, NY, AN, TY, HT, YU, and SI contributed to the material preparation, data collection and data analysis. $\mathrm{KI}$ and TM wrote the first draft of the manuscript. All authors commented on and approved previous versions of the manuscript.

\section{Acknowledgements}

This study was conducted for the patients at Seirei Hospice. The authors would like to thank all participants for taking part in this study.

\section{References}

1. Beller EM, van Driel ML, McGregor L, Truong S, Mitchell G. Palliative pharmacological sedation for terminally ill adults. Cochrane Database Syst Rev. 2015;1: CD010206.

2. Cherny NI, ESMO Guidelines Working Group (2014) ESMO Clinical Practice Guidelines for the management of refractory symptoms at the end of life and the use of palliative sedation. Ann Oncol. 25 Suppl 3:iii143-iii152. 
3. Cherny NI, Radbruch L, Board of the European Association for Palliative Care (2009) European Association for Palliative Care (EAPC) recommended framework for the use of sedation in palliative care. Palliat Med. 23:581-593.

$4 \llbracket$ Schildmann E, Schildmann J.Palliative sedation therapy: a systematic literature review and critical appraisal of available guidance on indication and decision making. J Palliat Med. 2014 May;17(5):60111.

5. Brinkkemper T, van Norel AM, Szadek KM, et al. The use of observational scales to monitor symptom control and depth of sedation in patients requiring palliative sedation: a systematic review. Palliat Med. 2013; 27: 54-67.

6खBelar A, Arantzamendi M, Payne S, et al. How to measure the effects and potential adverse events of palliative sedation? An integrative review. Palliat Med. 2021;35(2):295-314.

7囚Schildmann EK, Schildmann J, Kiesewetter I. Medication and monitoring in palliative sedation therapy: a systematic review and quality assessment of published guidelines. J Pain Symptom Manage. 2015;49(4):734-46. doi: 10.1016/j.jpainsymman.2014.08.013. Epub 2014 Sep 19.

8. Arantzamendi M, Belar A, Payne S, et al. Clinical Aspects of Palliative Sedation in Prospective Studies. A Systematic Review. J Pain Symptom Manage. 2020;20:30754-5.

9.Zhai Y, Cai S, Zhang Y. The Diagnostic Accuracy of Critical Care Pain Observation Tool (CPOT) in ICU Patients: A Systematic Review and Meta-Analysis. J Pain Symptom Manage. 2020;60(4):847-856.

10. Fedele S, Strasser S, Roulin MJ. Validation of the Critical Care Pain Observational Tool in Palliative Care. Pain Manag Nurs. 2020;21(4):360-364.

11. Arevalo JJ, Brinkkemper T, van der Heide A, et al. Palliative sedation: reliability and validity of sedation scales. J Pain Symptom Manage. 2012; 44: 704-14.

12.Maltoni M, Miccinesi G, Morino P, et al. Prospective observational Italian study on palliative sedation in two hospice settings $₫$ differences in casemixes and clinical care. Support Care Cancer. 2012囚20『2829-36.

13. Imai K, Morita T, Yokomichi N, et al. Efficacy of two types of palliative sedation therapy defined using intervention protocols $₫$ proportional vs. deep sedation. Support Care Cancer. 2018ه26ه1763-71.

14. Won YW, Chun HS, Seo M, et al. Clinical Patterns of Continuous and Intermittent Palliative Sedation in Patients With Terminal Cancer: A Descriptive, Observational Study. J Pain Symptom Manage. 2019 Jul;58(1):65-71.

15. Hui D, Frisbee-Hume S, Wilson A, et al. Effect of Lorazepam With Haloperidol vs Haloperidol Alone on Agitated Delirium in Patients With Advanced Cancer Receiving Palliative Care: A Randomized Clinical Trial. JAMA. 2017 Sep 19;318(11):1047-1056. 
16. Hui D, De La Rosa A, Wilson A, et al. Neuroleptic strategies for terminal agitation in patients with cancer and delirium at an acute palliative care unit: a single-centre, double-blind, parallel-group, randomised trial. Lancet Oncol. 2020 Jul;21(7):989-998.

17. Sessler CN, Gosnell MS, Grap MJ, et al. The Richmond Agitation-Sedation Scale: validity and reliability in adult intensive care unit patients. Am J Respir Crit Care Med. 2002;166:1338-44.

18. Ely EW, Truman B, Shintani A, et al. Monitoring sedation status over time in ICU patients: reliability and validity of the Richmond Agitation-Sedation Scale (RASS). JAMA. 2003; 289: 2983-91.

19. Benítez-Rosario MA, Castillo-Padrós M, Garrido-Bernet B, et al. Appropriateness and reliability testing of the modified Richmond Agitation-Sedation Scale in Spanish patients with advanced cancer. J Pain Symptom Manage. 2013; 45: 1112-9.

20.Imai K, Morita T, Mori M, et al. Development and linguistic validation of the Japanese version of the modified Richmond Agitation-Sedation Scale. Palliat Care Res. 2016;11:331-336. (in Japanese)

21. Steinhauser KE, Clipp EC, McNeilly M, et al. In search of a good death: observations of patients, families, and providers. Ann Intern Med. 2000 May 16;132(10):825-32.

22. Miyashita M, Sanjo M, Morita T, Hirai K, Uchitomi Y. Good death in cancer care: a nationwide quantitative study. Ann Oncol. 2007 Jun;18(6):1090-7.

23. Carson MG, Fitch MI, Vachon ML. Measuring patient outcomes in palliative care: a reliability and validity study of the Support Team Assessment Schedule. Palliat Med. 2000;14:25-36.

24. Miyashita M, Matoba K, Sasahara T, et al. Reliability and validity of the Japanese version of the Support Team Assessment Schedule (STAS-J). Palliat Support Care. 2004; 2:379-85

25. Hurlley AC, Volicer BJ, Hanrahan PA, et al. Assessment of discomfort in advanced Alzheimer patients. Res Nurs Health. 1992;15:369-377.

26. Pasman HR, Onwuteaka-Philipsen BD, Kriegsman DM, et al. Discomfort in nursing home patients with severe dementia in whom artificial nutrition and hydration is forgone. Arch Intern Med. 2005 Aug 822;165(15):1729-35.

27. van Deijck RH, Hasselaar JG, Verhagen SC, et al. Level of Discomfort Decreases After the Administration of Continuous Palliative Sedation: A Prospective Multicenter Study in Hospices and Palliative Care Units. J Pain Symptom Manage. 2016 Sep;52(3):361-9.

28. Snow AL, Weber JB, O'Malley KJ, et al. NOPPAIN: a nursing assistant-administered pain assessment instrument for use in dementia. Dement Geriatr Cogn Disord. 2004;17(3):240-6. 
29. Lukas A, Barber JB, Johnson P, Gibson SJ. Observer-rated pain assessment instruments improve both the detection of pain and the evaluation of pain intensity in people with dementia. Eur $\mathrm{J}$ Pain. 2013;17(10):1558-68.

30. Morita T, Tsunoda J, Inoue S, Chihara S, Oka K. Communication Capacity Scale and Agitation Distress Scale to measure the severity of delirium in terminally ill cancer patients: a validation study. Palliative medicine. 2001;15:197-206.

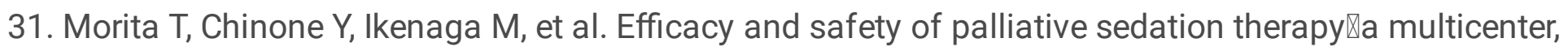
prospective, observational study conducted on specialized palliative care units in Japan. J Pain Symptom Manage. 2005®30®320-8.

32. Mercadante S, Intravaia G, Villari P, et al. Controlled sedation for refractory symptoms in dying patients. J Pain Symptom Manage. 2009ه37ه771-9.

33. Mercadante S, Porzio G, Valle A, et al. Home Care-Italy Group. Palliative sedation in patients with advanced cancer followed at home『a prospective study. J Pain Symptom Manage. 2014『47凶860-6.

34. Gélinas C, Puntillo K, Joffe A, Barr J. A validated approach to evaluating psychometric properties of pain assessment tools for use in nonverbal critically ill adults. Semin Respir Crit Care Med. 2013 ;34(2):153-68.

35. Zwakhalen S, Hamers J, Abu-Saad H, Berger M. Pain in elderly people with severe dementia: a systematic review of behavioural pain assessment tools. BMC Geriatr. 2006;6:3.

36. Fischer T, Hosie A, Luckett T, Agar M, Phillips J. Strategies for Pain Assessment in Adult Patients With Delirium: A Scoping Review. J Pain Symptom Manage. 2019;58(3):487-502.

37. Chookalayia H, Heidarzadeh $M$, Hassanpour-Darghah M, Aghamohammadi-Kalkhoran M, Karimollahi M. The Critical care Pain Observation Tool is reliable in non-agitated but not in agitated intubated patients. Intensive Crit Care Nurs. 2018;44:123-128.

38. Kanji S, MacPhee H, Singh A, et al. Validation of the Critical Care Pain Observation Tool in Critically III Patients With Delirium: A Prospective Cohort Study. Crit Care Med. 2016;44(5):943-7.

39. Campbell ML. Psychometric testing of a respiratory distress observation scale. J Palliat Med. 2008;11:44-50.

40. Zhuang Q, Yang GM, Neo SH, et al. Validity, reliability and diagnostic accuracy of the Respiratory Distress Observation Scale for assessment of dyspnea in adult palliative care patients. J Pain Symptom Manage. 2019;57:304-310.

\section{Figures}


1a) Intensity of physical symptoms measured by the STAS
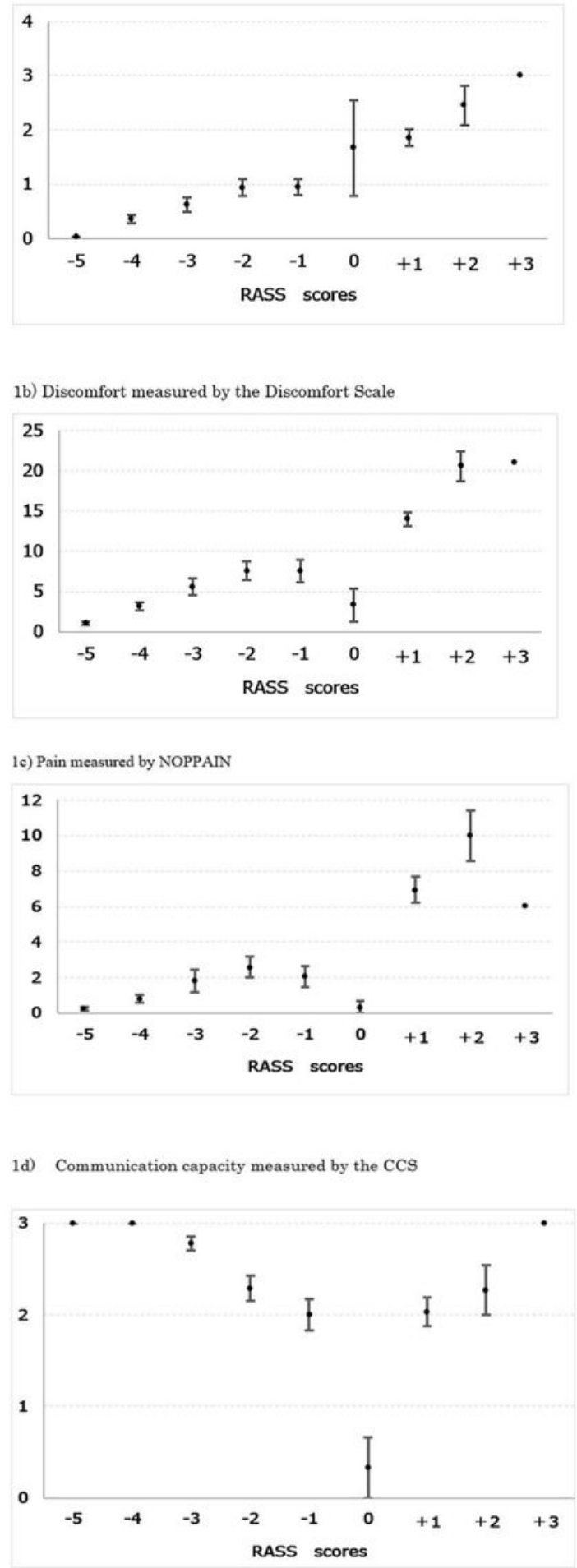

\section{Figure 1}

Difference in scores of the STAS, Discomfort Scale, NOPPAIN, CCS according to the RASS level. All P values for Pearson's correlation coefficients were $<0.001$. Error bars are standard errors. RASS: Richmond Agitation-Sedation Scale; STAS: Support Team Assessment Schedule, item 2 for any symptoms; Discomfort Scale: Discomfort Scale for Dementia of Alzheimer Type; NOPPAIN: Non-communicative patient's pain assessment Instrument; CCS: Communication Capacity Scale, item 4 